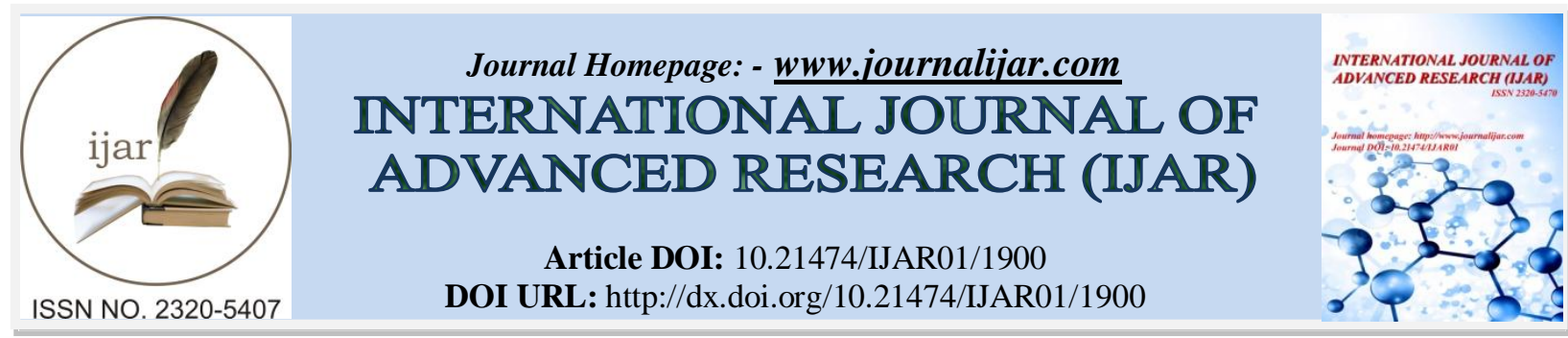

RESEARCH ARTICLE

\title{
A STUDY ABOUT THE KEY ELEMENTS OF TQS IN MANUFACTURED ITEMS.
}

Abdulkhalek M. Kadir.

Erbil Technical Engineering College, Erbil Polytechnic University (EPU)-Iraq.

\section{Manuscript Info}

Manuscript History

Received: 12 August 2016

Final Accepted: 22 September 2016

Published: October 2016

Key words:-

TQS, manufactured items, technical

features, maintenance.

\section{Abstract}

Technical quality system TQS is a system takes arranged technical tasks as technical features in order to improve quality of manufactured items in their design and operating. It refers to an integrated technical features related to 4 key elements such as availability, maintainability, recognition, and reliability. These key elements contain 4 groups of technical features connected to each element. They are vital features because they can result vital issues of design of manufactured items and high quality operating. They can give higher control and more efficient level. There are 33 technical features and 11 engineering equations in order to achieve quality pacifications of manufactured items by firms; each element holds several technical features with 1 to 5 engineering equations that can measure the values of the key element and detailed technical features.

As a new topic, the study of the key elements is regarded as a new approach for determining the values of mean time to repair or restoration, repair rate, recognition rate, and reliability value by related engineering equations.

As a new tactic, the study procedures have come via studying 4 key elements, determining technical features and the calculation of engineering equations, and classifying technical features to each one of elements.

Copy Right, IJAR, 2016,. All rights reserved.

\section{Nomenclature:-}

$R$ : value of reliability.

$e$ : natural log base.

$\lambda$ : failure rate.

$\lambda_{T}$ : failure rate for the total system (sum of the failure rates $\lambda_{n}$ ).

$t$ : time duration.

$A$ : fraction of total time in which the system is available.

$M T B F$ : mean time before failure.

MTTR: mean time to repair and return to the state before happening of failure.

$M T B F_{T}$ : reciprocal mean time before failure rate.

MTTRs: mean time to repair or restoration of the system level.

$\mu$ : repair rate. 
$D P$ : dynamic positioning system; in terms of failure risk costs and safety of operation, involves a human-machine system, indicate human factors in the reliability, it focuses on preventive maintenance and how failures can be reduced or postponed.

\section{Introduction and literature review:-}

An item is an individual article from a collection or a set [1], the items can be goods or products manufactured by production process in specialized manufacturing firms. An item can be final product or semi-final product. Manufacturing is the process of making products, it is the process of making wares from raw materials by hand or by using mechanical power and machinery, and it can be the process of producing something [2]. The manufacturing deals with large numbers of products. Thus others defined it as the business of goods in large numbers [3]. Manufactured items are those products that have been made from special raw materials by technical operations using machinery. The technical method is related to specializing manner in industrial or mechanical arts and applied sciences, it associates to techniques, particularly with applied industrial procedures [4], but the feature is the prominent characteristic and it can be the distinctive attribute of something [1], hence the technical features are subelements contain mathematical formulas linked with required key elements of TQS, and their values can be found by solving several equations of engineering. W. Edwards Deming defined good quality as a predictable degree of uniformity and dependability, and the quality is an outcome characteristic of a product or service provided to a customer [5]. Therefore, TQS is the quality system takes technical sides, and the technical sides would be arranged and controlled, thus, they will be as technical features in order to give objective by manufacturing items, in both stages of design and operating. Each item holds many technical features according to its use and the aims to which it was manufactured, these technical features are assumed as fest rules of technical specifications, but they enforced by quality standards provided to any type of items or any one of item's component. As a result TQS is assumed as measured system to determine values of technical features related to the manufactured items, and to achieve aims of efficient operations of the firms.

As literatures review, there are some previous studies such as:

- A study was achieved by H. Paul Barringer [6], he showed five elements such as effectiveness, availability, reliability, maintainability, and capability, then he gave inherent and operational availability. He presented also, some engineering equations in order to understand and calculate quantity examples in topics of failures, meantime failures, frequency of failures over a time interval, and technical arguments in production process.

- A study was presented by Bruno Sericola [7], he gave analysis of availability in the repairable computer system, and he presented new mathematical algorithm with simplified engineering equations. He determined also, values of equations and explained the expected interval analysis of availability element, the study affirmed a numerical example on fault-tolerant multiprocessor system, then considered two processes without repair task, but he extended in order to include computation's repair to obtain distribution of performability.

- Several researchers [8] were offered studies on new techniques in computing failures in order to enhance availability for the purpose of avoiding errors of memory, the aims were to known security results of the system and to enable system servers to successful operating with user's satisfaction.

\section{Methodology:-}

The methodology in this study consists of three steps that are assumed as complete study procedures on four key elements and finding values of technical features related to manufactured items by engineering equations, first step is study 4 key elements of TQS, second step is indicating their technical features and classifying them according to each element, third step is present mathematical formulas as engineering equations related to each element, fourth step contains several procedures such as analyzing of data on experiments done by previous studies, motives of the research, aims of the research, results and discussion, conclusions and recommendations.

\section{Motives of the research:-}

Why we choose TQS to be the basis of our study? To answer this question, we clarify reasons, and explain why this research try to determine and classify technical features related to each element, the motives are as follows:

- Key elements of TQS have been explained and their technical features classified, because they can bring high quality level for manufactured item.

- TQS has been chosen in this study, because many researchers were agreed that implementation of technical features can help firms to deliver their goals, in addition it can be applied as a technical system to assist other working systems that can improve quality level of items. 
- Manufactured items may be regarded as poor design and operating of items may be weak, therefore it is strongly required to find and classify technical features because they can achieve methods of dependable outcomes by manufactured items.

- Determining values of 4 key elements play great role to prevent inaccurate outcomes of manufactured items in stages of design and operating, because they can reduce failures, instability, and losses, and they can increase recognition, reliability, repair and maintenance rate, then lead to more success.

\section{Objectives of the research:-}

The research has some objectives, they are summarized as follows:

- Reviewing 4 key elements of TQS and related technical features of each element.

- Determining mathematical formulas as engineering equations associated to each element of TQS.

- Preparing an interrelation among quality level, key elements, and technical features in order to gain higher quality and recognized technical features.

- Explaining values results of availability, procedure of repairs, restoring, and failure rate, (physical, mechanical, and electrical) tests, contribution range by people and society, frequency of failures over a time interval, reliability, and maintenance range of manufactured items.

\section{Key elements of TQS:-}

Technical quality system TQS has several elements which enforce to built higher quality of items which manufactured by production system in a firm, the stand of the firm is calculated crucial factor, but 4 elements take more essential issue in related technical features especially on basis of associated mathematical formulas working as engineering equations. In all cases, in order to succeed TQS with their technical features, the firms should focus further on 4 key elements such as: availability, maintainability, recognition, and reliability [6\&7], because these elements represent many characteristics to improve quality level of manufactured items, these two concepts are complete each one others, as shown in Figure 1.

Elements of TQS would enforce quality of manufactured items by achieving results of technical features in related manufacturing firms, and when they assessed according to engineering equations in both design and operating in which they include several tasks of maintenance, repair time, repair rate, re-design, re-construction, logical calculation, respond to electronic and electric operations, respond to reliable modifications, respond to mechanical, environmental, and chemical operations, and other tasks. The triangle TQS includes three main parts such as: 4 key elements, 33 technical features, and manufactured items, as shown in Figure 2. These parts are more interrelated tasks when implemented in production process and together give higher quality to manufactured items, the triangle assumed as column enforce quality level.

\section{Key Elements of TQS}

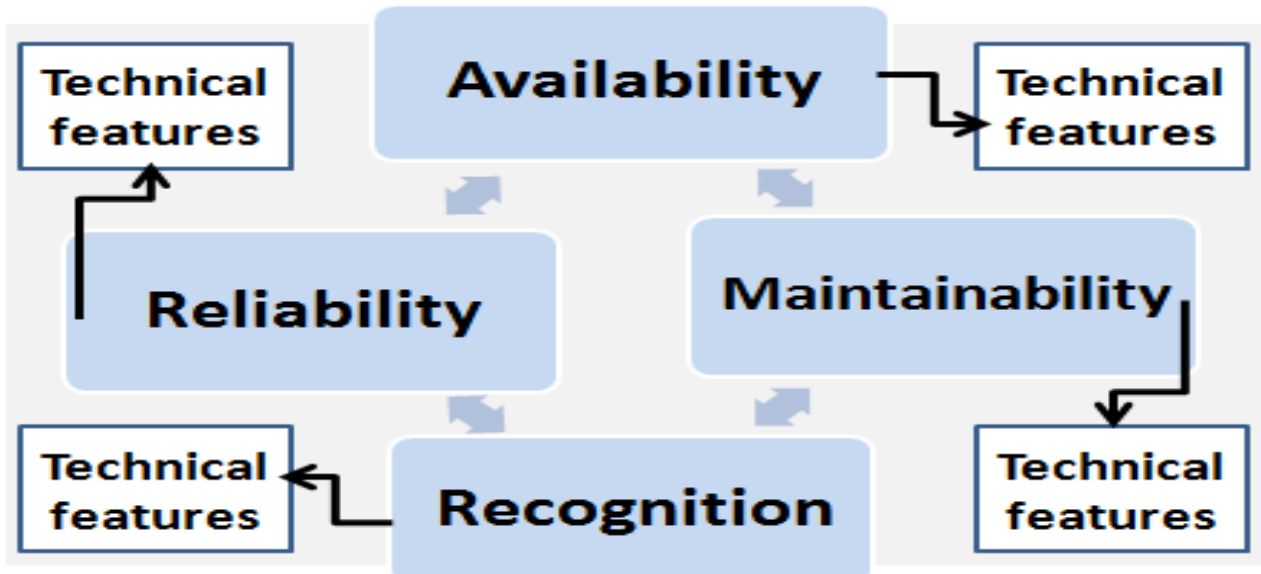

Figure 1: Key elements of TQS 
But technical features give active results and can be calculated according to some mathematical formulas which are in their processing assumed as engineering equations to item's design inside firms and item's operating outside firms or after manufacturing. Table 1 shows four key elements and their technical features.

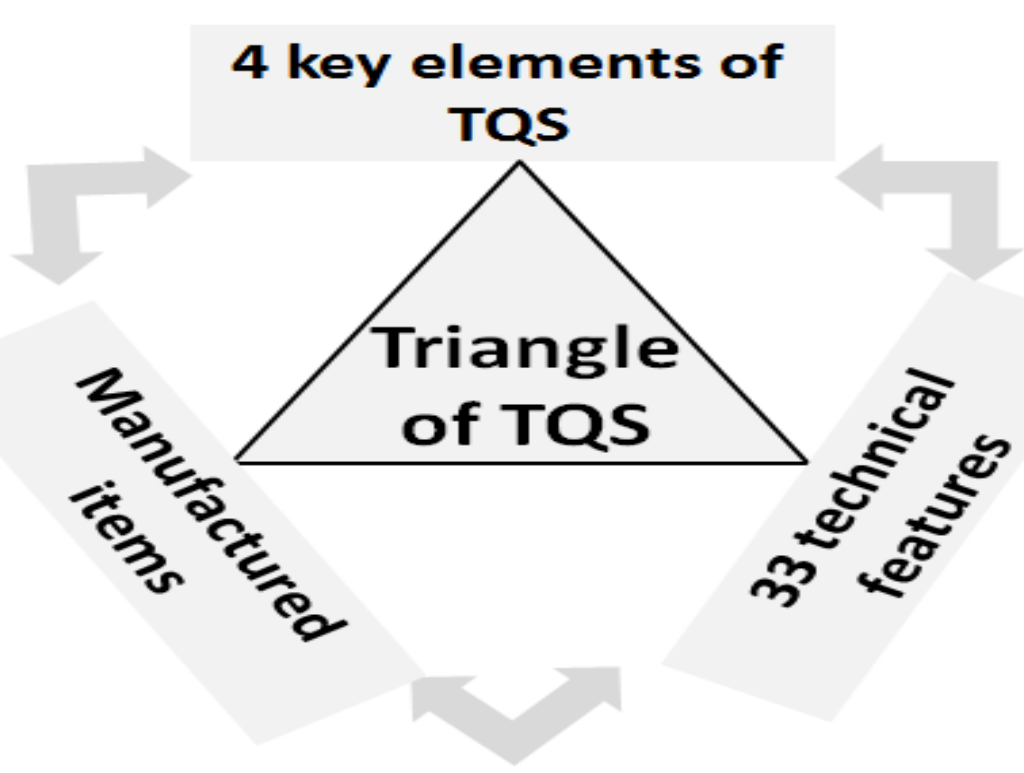

Figure 2: Triangle of TQS

Table 1:- 4 key elements of TQS and their technical features.

\begin{tabular}{|c|c|c|}
\hline & & Table 1: Technica \\
\hline & $\begin{array}{c}\text { Key elements } \\
\text { of TQS }\end{array}$ & Technical features \\
\hline 1 & Availability & $\begin{array}{l}\text { Mean time to restoration, up-time duration in operating of } \\
\text { items, value of capability to perform tasks in the } \\
\text { manufactured item, degree of respond by item tasks, range of } \\
\text { increasing time of operation, fraction of time for item's use } \\
\text { availability, fraction of shut down time during failures, failure } \\
\text { rate for the total system, reciprocal amount of failure rate. }\end{array}$ \\
\hline 2 & Maintainability & $\begin{array}{l}\text { Number of maintainability features in design of manufactured } \\
\text { items, duration of item's stoppage for the maintenance, real } \\
\text { time to repair, repair rate, volume of preventive and } \\
\text { corrective maintenance, number of used spare parts, amount } \\
\text { of physical, mechanical, and electrical tests, random variable } \\
\text { of time-to-repair, random variable of time-to-failure. }\end{array}$ \\
\hline 3 & Recognition & $\begin{array}{l}\text { Ratio of trust by customers, participation level in quality } \\
\text { system used in production process, degree of activating of } \\
\text { morals by designers, ratio of decision's contribution by } \\
\text { peoples and society on technical features, number of error } \\
\text { recognition samples, number of correct recognition samples. }\end{array}$ \\
\hline 4 & Reliability & $\begin{array}{l}\text { Failure rates and short exposure times, period for the item's } \\
\text { operating, distribution of the failure time, frequency of } \\
\text { failures over a time interval, failure in the free operation, } \\
\text { providing average of information to the computer pertaining } \\
\text { vessel's position, magnitude and direction of environmental } \\
\text { effects on vessel's position. }\end{array}$ \\
\hline
\end{tabular}




\section{Availability:-}

Availability is the first element of TQS, and it deals with duration of up-time of manufactured items operating. The availability assists to calculate mean time to repair, it can be a measure of how often the system is alive and well, and the availability is often expressed by the formula: (up-time) / (up-time + downtime) with many different variants [6], the up-time refers to a capability to perform the task by manufactured item and downtime refers to "not being" able to perform the task, here, the same state is true to process of item's maintenance, and how the item, or a component of the item, will agree the process. Thus in order to achieve any type of maintenance to manufactured items, the availability will deal with at least three main factors [6] such as:

1. Increasing time of item's operating until the failure is happened

2. Decreasing of downtime owing to repair items or other scheduled maintenance to repair

3. Accomplishing two factors above in a cost effective manner

Here, the element of availability is a wide range of delivered to an expected service, it receives expected value of manufactured items and how long it accepts maintenance or improvements. There are several engineering equations [9] in which the system of manufactured item is available for use and repair:

- Fraction of total time in which the system of item is available $A$

- Failure rate for the total system $\lambda_{T}$

- Mean time to repair or restoration MTTRs

\section{The equation of $A$ is as follows:-}

$$
A=\frac{M T B F}{M T B F+M T T R}
$$

Here $M T B F$ is mean time before failure, and MTTR is mean time to repair and returning to the state as almost before happening of failure. Thus in order to get more precise results, there is an equation that can put this state into standpoint related to series of subsystems of manufactured item in which limits the failure rate for the total system $\lambda_{T}$ which becomes the sum of the failure rates $\lambda_{n}$ and $\lambda_{T}$ will be equal to:

$$
\lambda_{T}=\lambda_{1}+\lambda_{2}+\lambda_{3}+\lambda_{4}+\lambda_{5}+\lambda_{6}+\lambda_{7}+\ldots .+\lambda_{n}
$$

The above equation can be formulated as:

$$
\frac{A}{M T B F_{T}}=\frac{A}{M T B F_{1}}+\frac{A}{M T B F_{2}}+\frac{A}{M T B F_{3}}+\frac{A}{M T B F_{4}}+\ldots \ldots+\frac{A}{M T B F_{n}}
$$

$M T B F_{T}$ is the reciprocal of mean time before failure rate, and others are for the subsystems.

According to this state, in stage of item's design process, considering of redundant of subsystems would be much effectual way to gain reliability improvement beside other modifications when exist, thus the mean time to repair or restoration MTTRs is calculated by following formula:

$$
\operatorname{MTTR}_{s}=\frac{\sum_{i=1}^{n} M T T R_{i} / M T B F_{i}}{\sum_{i=1}^{n} 1 / M T B F_{i}}
$$

Maintainability:-

Maintainability is the second element of TQS, and it takes great role to preserve every partial component of manufactured items in order to stay in working longer time in an efficient manner. The maintainability is the characteristic which should be added into stage of item's design calculation, also it takes large position on how use maintenance of manufactured items with well-organized quality level, as a result it deals with two cases of maintenance duration and how long it takes to achieve repairing. Thus, the maintainability considered as probability amount of maintenance used for manufactured item which is reflect predesigned time interval and technical features, it should be known that the maintainability deals with all types of maintenance such as types of preventive and corrective, but this rule is true when other resources calculated because they have obvious effect on outcome of 
maintenance, these resources are include: skill level of staff, spare parts, used facilities, inspection, physical, mechanical, and electrical test, etc. [9\&10].

In the maintainability the random variable is "time-to-repair" will be in same manner to random variable which is "time-to-failure" in the reliability, thus for system of item in which the times of repair are distributed exponentially,

There are several engineering equations [9\&6] in which the system of manufactured item ready of maintainability as follows:

- Equation of maintainability $M(t)$

- $\quad$ Repair rate $\mu$

The equation of maintainability is give by the formula:

$$
M(t)=1-e^{-\mu t}
$$

Where $\mu$ is repair rate and its value determined by the formula:

$$
\mu=\frac{1}{M T T R}
$$

MTTR is mean time to repair which its value determined practically by the firm.

There was an experimental study prepared by a research [6], it presented goal of maintainability stated in a firm with $90 \%$ likelihood which completed time of maintenance repair in 8 hours with a maximum repair time of 24 hours, and it required MTTR by 3.48 hours, the study mentioned goal of a stated maintainability which requires real control of three main downtime of manufactured items such as:

- The time of an active repair depends on function of design, training level, and personnel skills, theses are responsible and related to maintenance.

- The time of logistic which include time lost for supplying and replacement parts

- The time of administrative which include function of firm's structure

\section{Recognition:-}

Recognition is the third element of TQS, and it takes efficient role when activated by the employees or other concerned customers about manufactured items which are used by them and recognized. The level of trust from users of manufactured items is measured as crucial factor to raise quality of elements of TQS and related technical features, because without recognition and trust on TQS there will be difficult state to complete objectives, and TQS appears as lack operating system in the firms, because the recognition will allow everyone to do the best with individual features, the studies affirmed that the recognition enforces and calls for recognized technical features with other involved morals, values, honesty, sincerity and support with fact [11]. Four key elements of TQS will remain recognized by people and society because they strongly contribute in decision on values of technical features.

There was an experimental study prepared by a research about recognition effect comparison [12], it used 60 samples in order to represent tests, but every sample was trained and recognized in dynamical gesture sets. The results were analyzed and expressed in figures, and the figures showed the rate of individual recognition and average of extraction in gesture area, the study used an engineering equation of recognition in order to find recognition rate $\eta$ as computational equation:

$$
\eta=\frac{T R}{T R+F R}
$$

Where $\eta$ is recognition rate, $T R$ represents the number of correct recognition samples, and $F R$ represents the number of error recognition samples.

\section{Reliability:-}

Reliability is the fourth element of TQS, and it can give big role in revealing imperfection of manufactured items, because it deals with reducing frequency of failures over a time interval. The reliability contribute in generating confidence on the technical features which relate to manufactured items, therefore it considered as special 
characteristic which can add more satisfaction range from users of manufactured items, the reliability is a measure of the probability for failure-free operation during a given interval, i.e. it is a measure of success for a failure free operation [6]. The measuring method of reliability is the probability of an item to failure rate in relation with time; hence the reliability is expressed by the probability that the item will perform its required function under given conditions for a time interval [10].

There was an experimental study prepared by a research on dynamic positioning system DP which connected with the value of reliability [9], DP is considered decisive subsystem in terms of three aspects of: failure risk costs, operation's safety of a product, and probable failure of the system, this subsystem contains a computer controlled system to indicate results and make control, it used in oil and gas exploration industry but measured as humanmachine system in order to indicate human factor in reliability, it can focus on preventive maintenance and how failures can be reduced or postponed. An experimental study by an industry firm demonstrated bathtub curve as shown in Figure 3, it presents hypothetical failure rate versus time and shows three of decreasing, low constant, and increasing failure rates [13], but the results from the experimental study indicated early failure period and end failure period of used equipments, in addition, it has decreasing and increasing failure rate at the beginning and end of the process, also, it showed times of failures.

There are several engineering equations [6\&9] in which the system of manufactured item will take reliability in suitable level for further analysis and modeling by way of the exponential failure time distribution $R$ in which the system of manufactured item will take reliability in suitable level for further analysis and modeling by the way of exponential failure time distribution, then the system of manufactured item will be ready for working with reliable state. And the equation of $R$ is as follows:

$$
R=e^{-\lambda t}
$$

Where $R$ is the value of reliability, $e$ is the natural $\log$ base, $\lambda$ is the failure rate, and $t$ is time duration, but with low failure rates and short exposure times, the values for reliability are close to one, since failure rate $(\lambda)$ is the reciprocal of $M T B F$, then the formula of value of reliability will be equal to:

$$
\begin{aligned}
& \lambda=\frac{1}{M T B F} \\
& R=e^{\frac{-t}{M T B F}}
\end{aligned}
$$

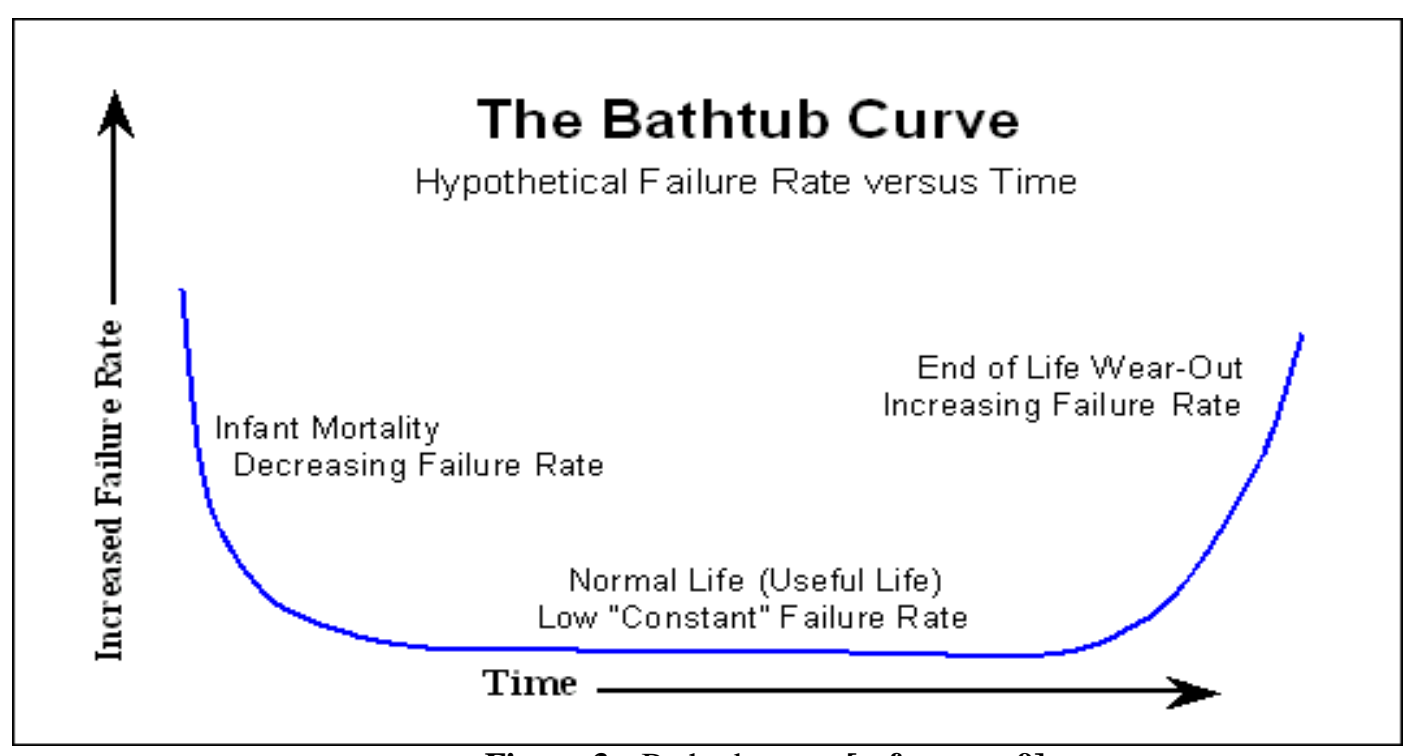

Figure 3:- Bathtub curve [reference: 9] 
Other study researched on mission time, because the defining of mission time is very important in reliability calculation to get valid answer result, an experimental study[6] achieved a mission time of one year with an equipment which has 30 year mean time to failure and gave a reliability of $96.72 \%$ which was the probability of successfully completing one year time interval without failure, and as the (reliability + unreliability $=1$ ), the probability for failure was $3.278 \%$, in addition the study was noticed that the high reliability for mission times of one year or more will require high inherent reliability and large mean times to failure, but with the fact that the inherent reliability is not achieved due to operating errors and maintenance errors.

\section{Data Analysis:-}

Availability, maintainability, recognition, and reliability are 4 elements of TQS, they are more important than others and regarded as key elements.

- Technical features are considered as sub-elements related to 4 key elements of TQS, they are assumed as dependable measurements for design values and operating level of manufactured items, they remain as measurable elements which working as a band in calculating values of each technical feature, as a result they give positive results to manufactured items.

- The sum of technical features is 33, each element of TQS has a number of technical features near to each other, therefore they take same importance and holds same share to complete quality of manufactured items.

- The sum of engineering equations is 11; each element of TQS has a number of engineering equations near to others, and has a suitable number in order to find values of technical features.

- Some technical features are vital and most necessary, they are related to features of: repair time, cost, stoppage, maintenance, recognition researching on side of human behaviors, mean time to repair or restoration for availability, repair rate for maintainability, recognition rate for maintainability, mission time related to preventive maintenance, value of reliability for reliability element.

\section{Results and Discussion:-}

Results:-

- TQS is a quality system that takes technical factors in order to be measured.

- TQS has many elements, four of them are vital such as: availability, maintainability, recognition, and reliability, they are assumed as key elements and regarded essential to manufactured items more than others.

- Availability has 9 technical features, maintainability has 11 , recognition has 6 , and reliability has 7 technical features.

- Availability has 5 mathematical formulas, maintainability has 2 , recognition has 1 , and reliability has 3 of them, they are engineering equations in their calculations.

- Elements give results of manufactured items as one total result, but each one affect on others, and for real quality result, all technical features should be calculated.

- Availability deals with the duration of up-time of operating of manufactured items, maintainability deals with duration of stoppage of the maintenance, recognition deals with the trust and involved morals, values, honesty, sincerity and support by customers or users of manufactured items, and reliability deals with reducing the frequency of failures over a time interval.

- The results are indicated that technical features are calculated as quality characteristics in items of design and operating after item's manufacturing in the firm.

- One year of a mission time of 30 year mean time to failure, the given reliability ratio will be equal to $96.72 \%$, and unreliability which names the probability for failure ration will be equal to $3.27 \%$.

\section{Discussion:-}

- The research is about manufactured items, because they occupies large share of industry sector, connects with people live and their safety, and have vital role in economy of the firms.

- TQS has many elements but 4 of them are regarded as key elements because they deal with manufactured items to achieve higher quality level.

- The elements hold a number of technical features between 7 to 11 and their numbers are near to each other, because they hold same vital role in design and operating of manufactured items, and this gives equality importance to each element towards quality improvement of manufactured items. 
- Each element takes between 1 to 5 engineering equations in order to find element's value, but they are related to design and operating which related to quality specifications level, and because this gives same manner of vital task related to calculation of the design and assure operating state.

- The effects of four key elements are as a band because they tend to achieve same objectives related to design and operating of manufactured items, and together, they can give results as integrated elements in order to reduce failures and losses, and they enforce maintenance of items.

- Element of availability deals with duration of up-time of operating of manufactured items and final value can be measured from engineering equations is mean time to repair or restoration, because it mixed design and operating together towards higher quality level. Element of maintainability deals with duration of stoppage of the maintenance of manufactured items and final value can be measured from engineering equations is repair rate, because it reduces maintenance time and cost in operating manufactured items. Element of recognition deals with trust and involved morals, values, honesty, sincerity and support by customers or users of manufactured items and final value can be measured from engineering equations is recognition rate for maintainability, because it increases trust and morals in design of items during production process and dealing with it by higher trust and morals by item's users. Element of reliability deals with reducing frequency of failures over a time interval of manufactured items and final value can be measured from engineering equations is value of reliability because it minimizes number of failures and defects in item's design and operable reliability of the components.

\section{Conclusions and Recommendations:-}

\section{Conclusions:-}

Technical quality system TQS is a technical system takes technical sides arranged by its technical features in order to improve quality of manufactured items in their designing and operating. TQS refers to an integrated technical features of 4 key elements which contain 4 groups of technical features related to each element, 4 key elements are vital because they result vital result in design and operating associated to manufactured items in the firm, they need more control and efficient to be at higher level of quality.

TQS becomes an effective theme because it holds quality competition between items and needed technical features, and it is not as alternative for managing problems nor gives complete solution for failures or defects, but it can discover the essence of failures, defects, and losses of time, in order to solve them in advance. Four key elements are availability, maintainability, recognition, and reliability, we understood that:

- The availability deals with the duration of up-time for operations in production process related to manufactured items, and it is a measure of how often the system is alive.

- The maintainability deals with duration of maintenance outages or how long it takes to achieve, it is calculated as the probability that an amount of any type of the maintenance of a manufactured item will be performed within predesigned time interval for limited technical procedure.

- The recognition deals with the ratio of trust by all employees, customers, and public responsible on related item and its role in peoples living, without recognition, the system will face problems that TQS cannot be effective and appear as lack system, and

- The reliability deals with reducing the frequency of failures over a time interval, it gives major contribution in generating the confidence on technical features of manufactured items and aspects of production process, it could be as a measure of success for a failure free operation.

There are33 technical features associated to 4 key elements, and there are 11 mathematical formulas which assumed as engineering equations to measure values of needed technical features. TQS is as quality system in technical sides for design and operating of items, and leads to improve quality level to manufactured items and reliability by users. The objectives of the research were to indicate key elements of TQS and study about technical features, present their engineering equations and to what aims they deal.

\section{Recommendations:-}

- Activating 4 key elements for item's manufacturing in design and operating stages, and record it in the policy of the firms.

- Determining the technical features related to each element in manufactured items and balancing them with quality specifications 
- Starting to implement TQS elements as a unit for all components of manufactured items in stages of design and operating.

\section{References:-}

1. http://www.oxforddictionaries.com/definition/english/feature

2. [http://www.merriam-webster.com/dictionary/manufacture

3. http://dictionary.cambridge.org/dictionary/english/manufacturing.

4. [http://www.dictionary.com/browse/feature

5. http://www.thecqi.org/The-CQI/What-is-quality/

6. H. Paul Barringer, P.E., Availability, Reliability, Maintainability, and Capability, Barringer \& Associates, Inc. Humble, TX, February 18, 1997, Beaumont, Texas,

7. Bruno Sericola, Availability Analysis of Repairable Computer Systems and Stationarity Detection, IEEE Transactions on computers, Vol. 48, No. 11. Nov. 1999.

8. Martin Rinard, Cristian Cadar, Daniel Dumitran, Daniel M. Roy, Enhancing Server Availability and Security Through Failure-Oblivious Computing, Tudor Leu, and William S. Beebee, Jr., Computer Science and Artificial Intelligence Laboratory, Massachusetts Institute of Technology, Cambridge, MA 02139.

9. Ali Ebrahimi, 2010, Effect analysis of Reliability, Availability, Maintainability and Safety (RAMS ) Parameters in design and operation of Dynamic Positioning (DP) systems in floating offshore structures, Master's Degree Project, KTH, Royal Institute of Technology, School of Industrial Engineering, Department Production Engineering and Management, Stockholm October 2010, page 69-71.

10. Reliability Engineering Theory and Practice A.Birolini Forth Edition

11. MASDER Kumar et. al., 2008,

12. Zhang Qiu-yu, Lv Lu, Zhang Mo-yi, Duan Hong-xiang and Lu Jun-chi, A Dynamic Gesture Trajectory Recognition Based on Key Frame Extraction and HMM, International Journal of Signal Processing, Image Processing and Pattern Recognition, Vol.8, No.6 (2015), pp.91-106,

13. Review of methods for demonstrating redundancy in dynamic positioning systems for the offshore industry. DNV Consulting for the Health and Safety Executive 2004 